\title{
Shaped by collisions
}

\author{
Melt rocks returned from the Moon date to a narrow interval of lunar bombardment about 4 billion \\ years ago. There is now evidence to show that this so-called Late Heavy Bombardment spanned the \\ entire Solar System.
}

Several hundred million years after its formation, the inner Solar System was pummelled by impactors. Ages of melted rocks sampled during the Apollo missions cluster in time, indicating a bombardment of cataclysmic proportions that produced the Moon's oldest and largest impact basins. But with a limited data set, the hypothesized Late Heavy Bombardment was initially controversial. Today, evidence of a Late Heavy Bombardment has been identified on the Earth and the other terrestrial planets, the asteroid belt, the icy moons of the outer Solar System, and even the gas giants.

The Late Heavy Bombardment, it turns out, is a Solar System story. It is tempting to focus on the uniqueness of each planet but the geosciences are rooted in a shared history with our planetary siblings. A web focus published with this issue (http:// go.nature.com/83PgXm) explores how the shared history of large ancient impacts shaped the geologic evolution of the terrestrial planets and moons.

The earliest Solar System was a violent backdrop for planet formation. Following the Sun's birth, the planets grew through collisions out of gas and dusty debris that littered the primordial accretionary disk. The planetary embryos then continued to grow through collisions and mergers with each other. Giant impacts literally shook these protoplanets to their cores - the proto-Earth was probably hit by a Mars-sized body to form the Earth and Moon $^{1}$, and the anomalously smooth northern hemisphere of Mars could be explained as a giant impact basin ${ }^{2}$. All this occurred relatively quickly, probably within 100 million years. As planet formation wound down and the Solar System became less cluttered, the cratering rates of large impactors should have declined to a trickle. But all was not serene.

Instead, the Moon was pummelled, its largest impact basins, as dated from Apollo samples, indicating unexpectedly high cratering rates 600 million years after Solar System formation. Furthermore, the oldest known impact materials on Earth have been linked to the tail end

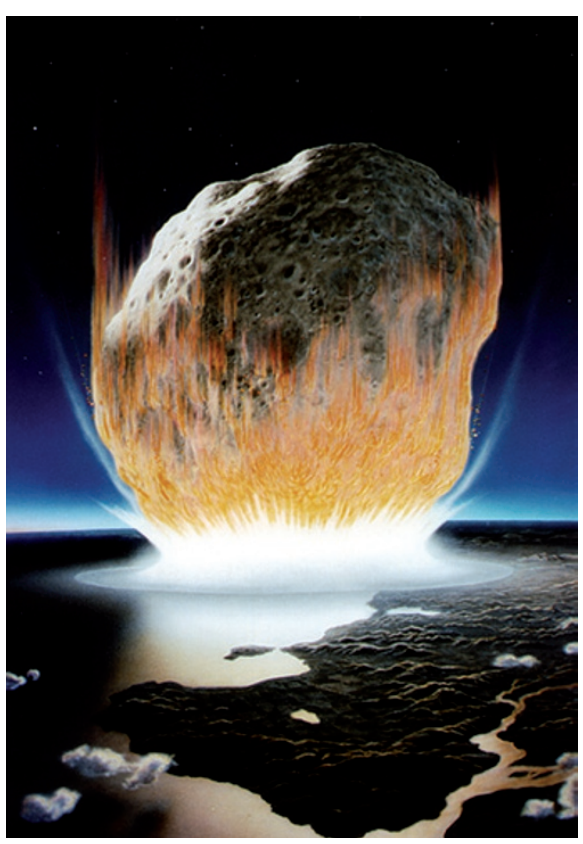

simulations suggest that the present-day orbital architecture of the outer Solar System can only be explained if the giant planets migrated after their formation into their present orbits, and that this migration occurred rapidly at about the same time as the Late Heavy Bombardment ${ }^{7}$. Giant planet migration would have disturbed a portion of the asteroid belt, sending a pulse of asteroids on to planet-crossing orbits. If the simulations are correct, then the terrestrial planets can blame big brothers Jupiter and Saturn and their orbital growing pains for the thumping they received 4 billion years ago.

The details of the Late Heavy Bombardment, such as its duration and magnitude, are still open for debate (page 520). Once a conundrum limited to the Moon, the Late Heavy Bombardment is now being tackled in studies that span the Solar System, as well as planetary science disciplines.

The shared violent history of ancient

of this violent interval ${ }^{3}$. Volcanism and cratering on Mercury ${ }^{4}$, geologic differences between Jupiter's icy moons ${ }^{5}$, and a period of unusually high velocity collisions within the asteroid belt itself ${ }^{6}$, have all been attributed to the Late Heavy Bombardment.

The geologic histories of planetary surfaces and the available samples of planetary materials point to a period of intense bombardment about 4 billion years ago. It is difficult to attribute such a lateoccurring bombardment to the remnants of planetary accretion. The impactors of the Late Heavy Bombardment must then have been delivered to the inner Solar System from a different source.

Perhaps the most important collision for understanding the Late Heavy Bombardment was not between planetary bodies, but between planetary scientists. Historically, planetary geology and planetary orbital dynamics were two fields that seldom intersected, but, as discussed in a Progress Article on page 520, the quest to make sense of the anomalous period of the Late Heavy Bombardment has brought these disparate fields together. Numerical impacts of the Earth and its planetary siblings influenced the evolution of planetary surfaces, interiors and even atmospheres. Intriguingly, and perhaps not entirely by coincidence, life on Earth started towards the end of the Late Heavy Bombardment. Perhaps the building blocks of life were delivered by some of these ancient impacts. Perhaps heat delivered by impacts supported long-lasting and habitable hydrothermal systems in which life could have evolved. And after life took root on Earth, perhaps early biological material was ejected from Earth by impacts and transported to the Moon ${ }^{8}$ or even, as the Commentary on page 510 explains, to a potentially habitable Mars. Thus life becomes a shared Solar System story as well.

\footnotetext{
References

1. Hartmann, W. K. \& Davis, D. R. Icarus 24, 504-515 (1975)

2. Melosh, H. J. Nature Geosci. 1, 412-414 (2008).

. Goldin, T. J. Nature Geosci. 5, 309 (2012)

4. Strom R. G. et al. Space Planet. Sci. 59, 1960-1967 (2011).

5. Barr, A. C. \& Canup, R. M. Nature Geosci. 3, 164-167 (2010).

Marchi, S. et al. Nature Geosci. 6, 303-307 (2013).

7. Gomes R., Levison, H. F., Tsiganis, T. \& Morbidelli, A. Nature 435, 466-469 (2005).

8. Asphaug, E. Nature Geosci. 6, 422-423 (2013).
} 